\title{
Investigation on Polarization Induced Electro-Optical Property of GaN LED Using TEM-EBIC Combined with Cathodoluminescence
}

\author{
M. H. Sheen, M. C. Lee, J. H. Lee and Y. W. Kim \\ Seoul National University, Department of Materials Science \& Engineering, Seoul 151-744, Korea
}

Strained InGaN layers grown on a c-plane sapphire is known to have strong spontaneous and piezoelectric polarization field, where the discontinuity of polarization at heterojunction is to induce bounded surface charges [1]. This polarization-related phenomenon can affect the internal electric field of active region and induce the quantum-confined stark effect (QCSE) at quantum well. QCSE results in the spatial separation of electrons and holes in the quantum well, blue shift, and increase of carrier density [2]. To reduce the polarization induced QCSE, efforts were made by growing GaN-InGaN on semi-polar and non-polar substrates [3]. In this study, we report the polarization induced electro-optical property of polar, semi-polar, and non-polar GaN-InGaN LED in nano-scale.

Electron-Beam Induced Current imaging (EBIC) in Transmission Electron Microscopy (TEM-EBIC) enables us to map the electrical response from region to region in nano-scale. When the EBIC is to combine with cathodoluminescence (CL) and microstructural analysis in transmission electron microscopy (TEM), emission characteristics can be directly correlated with the electrical properties and microstructure, possibly linked with the piezoelectric polarization and electrical junction property.

Electrical responses with electron beam were mapped with microstructure and the point-to-point mapping of local emission characteristics using home-built TEM-EBIC/CL holder (Fig. 1). The holder was equipped with liquid nitrogen cooling finger to minimize the carrier diffusion. Spectrum collection and the manipulation of the spectrum were carried out using home-built software from the panchromatic and monochromatic spectrum. Ion-milling system, instead of using focused ion beam, was used to avoid the artifacts of unintentional noise coming from the surface recombination current. First, we measured I-V curve of 3 TEM samples of the polar, semi-, and non-polar specimen to acquire the built-in voltage, breakdown voltage, and leakage current. And then, we acquired STEM-EDS on each specimen to identify microstructure and thickness of active region. Second, EBIC signal was obtained from each points of target analysis area under reverse bias condition, which enables us to estimate the depletion region width and the polarization field across on the $\mathrm{p}-\mathrm{n}$ junction. We also observed that the polarization filed was decreased from the sample grown on the c-plane to the one on the m-plane as expected. The minority carrier diffusion length showed dependency with polarization, which could arise from the space charge induced by polarization. CL spectra showed a tendency of blue-shift of emission in c-plane specimen as injection beam current increased, which might be originated from the bandgap fluctuation by the quantum-confined stark effect (QCSE) with strong polarization. CL spectra were obtained to confirm the polarization direction with the increase of reverse bias voltage. Peak shifts in CL might come from the cancellation of internal polarization with the external bias. Built-in voltage was also estimated with the polarization.

[1] Fabio Bernardini et al., Phys. Rev. B 56, R10024 (1997)

[2] Shigefusa F. Chichibu et al., Nature materials 5, 810 (2006)

[3] Pan et al., APEX 5, 062103 (2012) Number 42. 
[4] This research was supported by the Nano Material Technology Development Program(Green Nano Technology Development Program) through the National Research Foundation of Korea funded by the Ministry of Science, ICT \& Future Planning (2011-0019984).

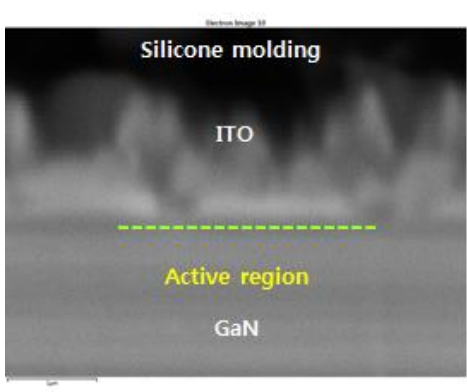

$<$ SE image $>$
Ga L $\alpha 1 \_2$

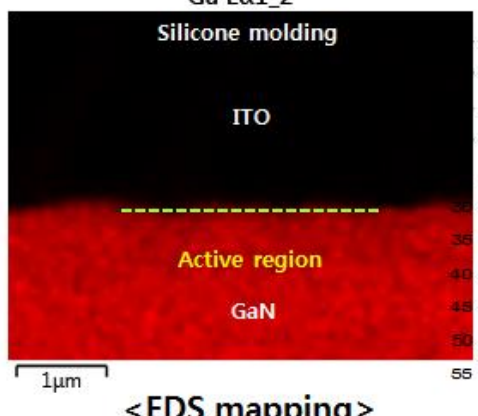

$<$ EDS mapping >

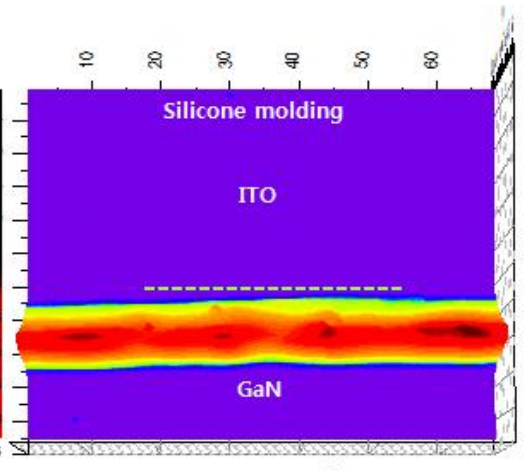

$<$ EBIC mapping >

Figure 1. EBIC and EDS mapping of GaN LED active region. 